\title{
Inhibition of DKC1 Induces Telomere-related \\ Senescence and Apoptosis in Lung Adenocarcinoma
}

\section{Guangyan Kan}

State Key Laboratory of Oncology in South China, Collaborative Innovation Center for Cancer Medicine, Sun Yat-sen University Cancer Center, Guangzhou, Guangdong 510060, P.R. China

\section{Ziyang Wang}

State Key Laboratory of Oncology in South China, Collaborative Innovation Center for Cancer Medicine, Sun Yat-sen University Cancer Center, Guangzhou, Guangdong 510060, P.R. China

\section{Chunjie Sheng}

State Key Laboratory of Oncology in South China, Collaborative Innovation Center for Cancer Medicine, Sun Yat-sen University Cancer Center, Guangzhou, Guangdong 510060, P.R. China

\section{Chen Yao}

State Key Laboratory of Oncology in South China, Collaborative Innovation Center for Cancer Medicine, Sun Yat-sen University Cancer Center, Guangzhou, Guangdong 510060, P.R. China

\section{Yizhi Mao}

State Key Laboratory of Oncology in South China, Collaborative Innovation Center for Cancer Medicine, Sun Yat-sen University Cancer Center, Guangzhou, Guangdong 510060, P.R. China

\section{Shuai Chen ( $\nabla$ chenshuai@sysucc.org.cn )}

State Key Laboratory of Oncology in South China, Collaborative Innovation Center for Cancer Medicine, Sun Yat-sen University Cancer Center, Guangzhou, Guangdong 510060, P.R. China https://orcid.org/0000-0003-1280-018X

\section{Research}

Keywords: lung adenocarcinoma, DKC1, telomere, cell senescence

Posted Date: October 26th, 2020

DOI: https://doi.org/10.21203/rs.3.rs-96223/v1

License: (c) (i) This work is licensed under a Creative Commons Attribution 4.0 International License. Read Full License 
Version of Record: A version of this preprint was published at Journal of Translational Medicine on April 20th, 2021. See the published version at https://doi.org/10.1186/s12967-021-02827-0. 


\section{Abstract}

\section{Background}

Lung cancer is one the most widely spread cancers in the world and half of the non-small cell lung cancers are lung adenocarcinoma (LUAD). Although there were several drugs been approved for LUAD therapy, a large portion of LUAD still can not be successfully treated due to lack of available therapeutic targets. Here, we investigated the oncogenic roles of DKC1 in LUAD and explored the potential mechanism and the possibility for LUAD therapy.

\section{Methods}

We analyzed Gene Expression Omnibus (GEO) and The Cancer Genome Atlas Program (TCGA) database and tissue microarray of LUAD. The expression of DKC1 and its correlation with prognosis were examined. In addition, Loss- and gain-of-function assays were used for oncogenic function of DKC1 both in vitro and in vivo.

\section{Results}

DKC1 is overexpressed in LUAD compared with normal tissues. High expression of DKC1 predicts the poor overall survival. Knockdown DKC1 in LUAD cell lines induced G1 phase arrest and inhibits cell proliferation. Ectopic expression of DKC1 could rescue the growth of LUAD cell lines. The abundance of $D K C 1$ is positively correlated with TERC and TERT levels. DKC1 downregulation caused decreased TERC expression, reduced telomerase activity and shorten telomere, and thus eventually led to cell senescence and apoptosis.

\section{Conclusions}

Our results show that high DKC1 expression indicates poor prognosis and DKC1 downregulation could induce telomere-related cell senescence and apoptosis. This study suggests that DKC1 could serve as a candidate diagnostic biomarker and therapeutic target for LUAD.

\section{Background}

Lung cancer is the leading cause of cancer death worldwide[1]. Lung adenocarcinoma (LUAD) is currently the most common type of lung cancer, which counts for about half of all non-small cell lung cancers[2]. In the past decades, there are several diver genes for LUAD having been identified and targeted therapies against them have achieved remarkable success. Inhibitors against epidermal growth factor receptor (EGFR), anaplastic lymphoma kinase (ALK), BRAF V600E mutation and MET Exon 14 skipping have been approved by FDA for precision treatment of $\operatorname{LUAD}[3,4]$. Despite these progression, there is still large portion of LUAD without available therapeutic target. 
The dyskerin pseudouridine synthase 1 (DKC1) gene which encodes dyskerin was first identified in dyskeratosis congenita (DC) $[5,6]$. Dyskerin is a component of telomerase ribonucleoprotein that associates with three highly conserved proteins (NOP10, NHP2 and GAR1) and binds directly to telomerase RNA component (TERC) to maintain TERC stability, telomerase stability and telomere length $[7,8]$. Recent reports showed that dysregulated expression of DKC1 in various human cancers alters cancer cell growth or metastasis and is associated with patient prognosis [9-11]. In addition, the antibiotics pyrazofurin (PF) was identified as a potent DKC1 inhibitor[12]. However, whether DKC1 promotes cancer cell growth in lung cancer and whether DKC1's oncogenic function is dependent on the regulation of telomere remains largely unknown.

In this study, we found that compared with normal tissues, DKC1 has higher expression in LUAD. The abnormal upregulation of DKC1 is associated with poorer prognosis of LUAD patients. TERC and TERT expression are positively correlated with $D K C 1$ level. In addition, we also identified that DKC1 downregulation decreases LUAD proliferation via telomere-related senescence and apoptosis.

\section{Methods}

\section{Cell culturing and human tissue microarray}

A549, PC-9 and NCl-H1299 cells were obtained from American Type Culture Collection (ATCC) and maintained by following the culture instructions of ATCC. Human lung adenocarcinoma tissue microarray (HLugA020PG01) was obtained from Shanghai Outdo Biotech Company.

\section{Generation of stable knockdown or overexpression cells}

shRNA for DKC1 (shDKC1-1,-2) was cloned into lentiviral pLK0.1 constructs (Sigma-Aldrich). The sequence of the shDKC1-1 is CCGGGCTCAGTGAAATGCTGTAGAACTCGA

GTTCTACAGCATTTCACTGAGCTTTTTG and the sequence of the shDKC1-2 is CCGGTAT

GTTGACTACAGTGAGTCTCTCGAGAGACTCACTGTAGTCAACATATTTTTT. The coding sequence of DKC1 was cloned into lentiviral pCDH-EF1-T2A-zeocin vector (System Bioscience). The virus particles were produced according to the lentivirus packaging protocol of Addgene. After shRNA lentivirus infection of lung adenocarcinoma cells lines (A549 and PC-9), puromycin (Thermo Fisher Scientific) selection was applied for at least 7 days to obtain stable knockdown cells. The overexpression lentivirus infected DKC1 stable knockdown cells or NCl-H1299 cells were selected with zeocin (Thermo Fisher Scientific) for at least 7 days to obtain ectopically expressed cells.

\section{Cell proliferation and cell cycle assay}

For cell growth curve, 1,500 cells was plated in one well of 96-well plate with at least 3 repeats for each cell and Cell Counting Kit-8 (CCK-8, Dojindo) were used to do continuous detection for 4-5 days. For colony formation assay, 1,000 cells were plated in one well of 6-well plate with at least 3 repeats for each cell and 7-9 days later were stained with crystal violet. Image $\mathrm{J}$ was used for colony number counting. For 
pyrazofurin (Sigma-Aldrich) affecting cell growth, 1,500 cells was plated in one well of 96-well plate with at least 3 repeat for each condition and the next day pyrazofurin or vehicle was added. CCK-8 was used to do the continuous detection for 4-5days. One million of DKC1 stable knockdown A549 and PC-9 cells or control cells were harvested for propidium iodide (PI) staining and cell cycle assay.

\section{Western blot}

$30 \mu \mathrm{g}$ protein were subjected to $12 \%$ SDS-PAGE. The following antibodies were used for western blot: DKC1 (sc-373956, Santa Cruz Biotechnology), P21 (sc-6246, Santa Cruz Biotechnology), yH2A.X (\#2595, Cell Signaling Technology).

\section{Quantitative PCR-based Telomerase Repeated Amplification Protocol}

The Telomerase Repeated Amplification Protocol (TRAP) is one sensitive telomerase activity detection assay. The quantitative PCR-based TRAP assay (qTRAP) is a more convenient measurement methods[13]. DKC1 stable knockdown A549 and PC-9 cells or control cells were harvested and 5000, 500 and 50 cells were used to assess the telomerase activity. The products of qTRAP (5000 cells) were visualized on a $10 \%$ non-denaturing acrylamide gel.

\section{Telomere length measurements}

The mean telomere length was measured by a quantitative PCR-based technique[14] in DKC1 knockdown A549 and PC-9 cells. The mean telomere length was expressed as a ratio (T/S) of telomere repeat length $(T)$ to the copy number of a single copy gene (S, $\beta$-Globin). The followings were the sequence of the PCR primers.

T1: 5'-ACACTAAGGTTTGGGTTTGGGTTTG GGTTTGGGTTAGTGT-3'

T2: 5'-TGTTAGGTATCC CTATCCCTATCCCTATCCCTATCCCTAACA-3'

S1: 5'-CGGCGGCGGGCGGCGCGGGCTGGGCGGcttcatccacgttcaccttg-3'

S2: 5'-GCCCGGCCCGCCGCGCCCGTCCCGCCGgaggagaagtctgccgtt-3'

\section{Cytochemical detection of senescence-associated- $\beta$-galactosidase activity}

DKC1 stable knockdown A549 and PC-9 cells or control cells (0.5 million) were plated in 6-well plate. Chromogenic $\beta$-gal substrate X-gal (C0602, Beyotime) were used to stain the fixed cells for 8 hours. The cells were washed with phosphate-buffered saline (PBS) and imaged by bright field microscopy.

\section{Subcutaneous Xenograft}

BABL/c female nude mice were obtained from Beijing Vital River Laboratory Animal Technology Company (Beijing). 1.5 million cells were injected to 5-6 weeks-old mice subcutaneously. When the tumors were detectable, tumor length and width were measured every 3 days. Tumor volume was 
calculated by using the following formula: tumor volume $=\left(\right.$ length $\times$ width $\left.^{2}\right) / 2$. After 30 days of injection, the tumor was removed and sent for immunohistochemistry analysis.

\section{Immunohistochemistry (IHC) and survival analysis}

The following antibodies were used for immunohistochemistry: DKC1 (sc-373956, Santa Cruz Biotechnology), Ki67 (550609, BD Biosciences) and YH2A.X (\#2595, Cell Signaling Technology). The DKC1 expression in human lung adenocarcinoma tissues was interpreted independently by two pathologists. Two characteristics were used for scoring the expression of DKC1 in slices: overall stain intensity (with possible values ranging from 0 to 3 ) and a score representing the percentage of tumor cells that were stained $(1,0-25 \% ; 2,25-50 \% ; 3,50-75 \%$ and $4,>75 \%)$. An IHC score was then calculated by multiplying the values of the two characteristics. Based on IHC score, patients were divided into two groups: high DKC1 expression (IHC score 4) and low DKC1 expression (IHC score<4). Overall survival (OS) were estimated using the Kaplan-Meier method, and the log-rank test was used for statistical analysis.

\section{Quantitative RT-PCR}

The qRT-PCR were performed and normalized by the expression level of GAPDH as an internal control. The sequences of the primers for qRT-PCR were listed as follows.

DKC1 F: 5'-ATGGCGGATGCGGAAGTAAT-3'

DKC1 R: 5'-CCACTGAGACGTGTCCAACT-3'

TERC F: 5'-ACCCTAACTGAGAAGGGCGTA-3'

TERC R: 5'-AATGAACGGTGGAAGGCGG-3'

GAPDH F: 5'-GAAGGTGAAGGTCGGAGTC-3'

GAPDH R: 5'-GAAGATGGTGATGGGATTTC-3'

\section{Statistics}

GraphPad Prism was used to conduct the statistical analysis for most of the data unless indicated otherwise. Statistical significance was assessed by ANOVA except when stated otherwise.

\section{Results}

\section{High expression of DKC1 in LUAD predicts poor prognosis}

We firstly examined DKC1 expression in LUAD tissues. The Gene Expression Omnibus (GEO) dataset (GSE75037) showed that compared with normal tissues, the 83 paired LUAD tissues show higher expression of $D K C 1$ (Fig. 1a). In addition, The Cancer Genome Atlas (TCGA) databases also revealed 
higher expression of $D K C 1$ in LUAD tissues ( $\mathrm{N}=483$ ) (Fig. 1b). The higher expression of DKC1 from TCGA databases had worse overall survival (OS) (Fig. 1C). To confirm the prognostic significance of high expression of DKC1 in LUAD patients, immunohistochemical (IHC) staining of DKC1 was performed on 84 LUAD tissues and 68 compared normal tissues. There is almost no DKC1 expression in normal tissues, and higher DKC1 expression predicted worse OS (Fig. $1 \mathrm{f}$ and 1g). Besides, both TERC and TERT (telomerase reverse transcriptase) expression positively correlated with $D K C 1$ expression in TCGA datasets (Fig. 1d and 1e).

\section{DKC1 accelerates LUAD cell proliferation}

To investigate the function of DKC1 in LUAD, lentiviral transduction of DKC1 shRNAs were used to reduce the DKC1 expression in two LUAD cell lines (A549 and PC-9). Both shRNAs for DKC1 could efficiently knockdown DKC1 protein levels in A549 and PC-9 (Fig. 2a). A549 and PC-9 with DKC1 silencing show decreased cell growth curve by CCK8 assay (Fig. 2b) and reduced colony number (Fig. 2c). In addition, knockdown DKC1 leads to cell cycle arrest at G1 phase in A549 and PC-9 cells (Fig. 2d). DKC1 inhibitor pyrazofurin (PF) also deceases cell growth curve and colony number in A549 and PC-9 cell lines (Fig. 2e and $2 f)$.

Next, we performed lentiviral transduction of DKC1 in NCl-H1299 cells without endogenous DKC1 expression (Fig. 3a). Overexpression of DKC1 in NCl-H1299 cells increase colony number (Fig. 3b). Furthermore, the successfully re-expressed DKC1 in DKC1 downregulated A549 cells (Fig. 3c) could restore the cell growth and colony formation (Fig. 3d and 3e). Taken together, DKC1 accelerates cell cycle progression and promotes cell proliferation in LUAD cell lines.

\section{DKC1 downregulation induces telomere-related senescence and apoptosis}

Furthermore, we investigated the mechanism of DKC1 to promote cell proliferation. As DKC1 is one component of telomerase ribonucleoprotein, we firstly examined the effects of DKC1 downregulation on telomere in LUAD cell lines. Both the level of TERC and telomerase activity are significantly decreased in DKC1 silenced A549 and PC-9 cells (Fig. 4a and 4b). And DKC1 downregulated A549 and PC-9 cells revealed reduced length of telomere (Fig. 4c). In addition, we found that telomere length reduce induced by DKC1 knockdown lead to cell senescence and apoptosis (Fig. $4 \mathrm{~d}$ and $4 \mathrm{e}$ ). There are more senescence associated $\beta$-galactosidase positive cells in DKC1 silenced A549 and PC-9 cells (Fig. 4d). The expression of cell senescence and apoptosis marker p21 and DNA damage marker YH2A.X are higher in A549 and PC-9 cells with DKC1 downregulation compared to control cells (Fig. 4e). These data suggest that DKC1 downregulation inhibits LUAD cell proliferation by inducing telomere-related cell senescence and apoptosis.

\section{DKC1 promotes LUAD cell proliferation in vivo}

Subsequently, we validated the function of DKC1 in xenograft mice model. DKC1 silenced PC-9 cells and control cells were subcutaneously inoculated into the BALB/c nude mice. The tumor volume and weight 
were monitored and measured (Fig. 5a and $5 b$ ). The tumor growth is much slower in DKC1 downregulated PC-9 cells compared to control cells (Fig. 5b). The detectable tumor for DKC1 silenced PC9 cells emerged at day 18, which is much later than control cells (day 9) (Fig. 5b). And at the end of the experiment, the tumor weight in DKC1 knockdown PC-9 cell group is lighter than the control group (Fig. 5a and $5 \mathrm{c}$ ). The DKC1 knockdown tumor cells show less proliferation marker Ki67 positive cells compared to control cells (Fig. 5d). At the meantime, there are more DNA damage marker $\mathrm{YH} 2 \mathrm{~A}$.X positive cells in DKC1 knockdown tumors (Fig. 5d). Taken together, DKC1 accelerates LUAD cell proliferation in vitro and in vivo. Targeted inhibition of DKC1 in LUAD cells resulted in telomere shortening, cell senescence and apoptosis.

\section{Discussion}

Telomeres play a critical role in preventing loss of essential genetic information during replication and distinguishing chromosome ends from DNA double-strand breaks, and ensure genome stability and integrity. Telomerase is a reverse transcriptase which adds telomeric repeats onto chromosome ends to maintain telomere length. Thus, telomerase activity needs to be carefully controlled. More than $90 \%$ of human tumors including lung cancer exhibited reactivation of telomerase and short telomeres[15-17]. As there is almost no detectable telomerase activity in most normal tissues and short telomere in tumor tissues, inhibition of telomerase is an attractive strategy for cancer therapy. Several approaches have been developed to inhibit telomerase, including antisense oligodeoxynucleotides, hammerhead ribozymes, catalytic (hTERT) component and small molecule inhibitors [18, 19]. Some of them have entered the phase I and II clinical trials [18, 19]. Imetelstat (GRN163L) acting as a direct telomerase RNA template antagonist has entered phase II clinical trials in advanced and metastatic non-small cell lung cancer (NSCLC)[20-22]. The efficacy of anti-telomerase therapy depends on the initial shortest telomere length which dictates the onset of telomere dysfunction[23]. As tumor cells exhibit short telomere and very little telomerase could maintain the shortest telomeres[24], it is indicating that highly potent inhibitors are needed.

In this study, we found that high DKC1 expression is associated with LUAD progression and indicates poor prognosis. DKC1 downregulation inhibits telomerase activity, induces telomere length shorten in lung cancer cells. Targeted inhibition of DKC1 increased telomere-related senescence and apoptosis both in vitro and in vivo. Our research indicated that $\mathrm{DKC1}$ is a promising target for telomerase-based therapies in lung cancer.

\section{Conclusions}

In conclusion, our data suggest that high expression of DKC1 could act as a candidate marker for diagnosis and therapy target in lung adenocarcinoma by inducing telomere-related cell senescence and apoptosis.

\section{Abbreviations}


DKC1: Dyskerin pseudouridine synthase 1

LUAD: Lung adenocarcinoma

TERC: Telomerase RNA component

TERT: Telomerase reverse transcriptase

PF: Pyrazofurin

TRAP: Telomerase repeated amplification protocol

\section{Declarations}

\section{Availability of data and materials}

Raw data of this study have been deposited in Research Data Deposit database

(http://www.researchdata.org.cn), and the accession number will be available upon acceptance.

\section{Funding}

This project was supported by grants from the Sci-Tech Project Foundation of Guangzhou City (201803010003).

\section{Authors' contributions}

G.K. performed most of the experiments and analyses. G.K., Z.W. and C.Y. performed the mouse experiments. C.S. and Y.M. provided technical assistance. S.C. conceived the study. S.C. and G.K. wrote the manuscript.

\section{Acknowledgements}

We thank Dr. Tianpeng Zhang helps in Telomerase Repeated Amplification Protocol analyses.

\section{Ethics approval and consent to participate}

The study with human clinical samples was approved by the Shanghai Outdo Biotech Company institutional review board (YB M-05-02). All animal studies were carried out according to the National Institute of Health Guide for the Care and Use of Laboratory Animals with the approval of Sun Yat-Sen University Cancer Center Institutional Animal Care and Use Committee.

\section{Consent for publication}

The authors declare no conflicts of interest. All authors read and approved the final manuscript.

\section{Competing interests}


The authors declare no competing financial interests.

\section{References}

1. Bray F, Ferlay J, Soerjomataram I, Siegel RL, Torre LA, Jemal A. Global cancer statistics 2018 : GLOBOCAN estimates of incidence and mortality worldwide for 36 cancers in 185 countries. CA Cancer J Clin. 2018;68:394-424.

2. Herbst RS, Morgensztern D, Boshoff $C$. The biology and management of non-small cell lung cancer. Nature. 2018;553:446-54.

3. Tulpule A, Bivona TG. Acquired Resistance in Lung Cancer. Annual Review of Cancer Biology. 2020;4:279-97.

4. Coudray N, Ocampo PS, Sakellaropoulos T, Narula N, Snuderl M, Fenyö D, et al. Classification and mutation prediction from non-small cell lung cancer histopathology images using deep learning. Nat Med. 2018;24:1559-67.

5. Nelson ND, Bertuch AA. Dyskeratosis congenita as a disorder of telomere maintenance. Mutation research. 2012;730:43-51.

6. Mason PJ, Bessler M. The genetics of dyskeratosis congenita. Cancer genetics. 2011;204:635-45.

7. Wong JMY, Collins K. Telomerase RNA level limits telomere maintenance in X-linked dyskeratosis congenita. Genes \& development. 2006;20:2848-58.

8. Caton EA, Kelly EK, Kamalampeta R, Kothe U. Efficient RNA pseudouridylation by eukaryotic H/ACA ribonucleoproteins requires high affinity binding and correct positioning of guide RNA. Nucleic Acids Res. 2018;46:905-16.

9. Hou P, Shi P, Jiang T, Yin H, Chu S, Shi M, et al. DKC1 enhances angiogenesis by promoting HIF1alpha transcription and facilitates metastasis in colorectal cancer. $\mathrm{Br} \mathrm{J}$ Cancer. 2019.

10. Miao FA, Chu K, Chen HR, Zhang M, Shi PC, Bai J, et al. Increased DKC1 expression in glioma and its significance in tumor cell proliferation, migration and invasion. Invest New Drugs. 2019.

11. Ko E, Kim JS, Ju S, Seo HW, Chang Y, Kang JA, et al. Oxidatively modified protein-disulfide isomeraseassociated 3 promotes dyskerin pseudouridine synthase 1-mediated malignancy and survival of hepatocellular carcinoma cells. Hepatology. 2018.

12. Rocchi L, Barbosa AJ, Onofrillo C, Del Rio A, Montanaro L. Inhibition of human dyskerin as a new approach to target ribosome biogenesis. PLoS One. 2014;9.

13. Jiang S, Tang M, Xin H, Huang J. Assessing Telomerase Activities in Mammalian Cells Using the Quantitative PCR-Based Telomeric Repeat Amplification Protocol (qTRAP). Methods Mol Biol. 2017;1587:95-101.

14. Cawthon RM. Telomere length measurement by a novel monochrome multiplex quantitative PCR method. Nucleic Acids Res. 2009;37.

15. Fernandez-Garcia I, Ortiz-de-Solorzano C, Montuenga LM. Telomeres and Telomerase in Lung Cancer. Journal of Thoracic Oncology. 2008;3:1085-8. 
16. Xu L, Li S, Stohr BA. The role of telomere biology in cancer. Annu Rev Pathol. 2013;8:49-78.

17. Günes C, Rudolph KL. The Role of Telomeres in Stem Cells and Cancer. Cell. 2013;152:390-3.

18. White LK, Wright WE, Shay JW. Telomerase inhibitors. Trends in Biotechnology. 2001;19:114-20.

19. Ruden M, Puri N. Novel anticancer therapeutics targeting telomerase. Cancer Treatment Reviews. 2013;39:444-56.

20. Mavroudis D, Bolonakis I, Cornet S, Myllaki G, Kanellou P, Kotsakis A, et al. A phase I study of the optimized cryptic peptide TERT(572y) in patients with advanced malignancies. Oncology. 2006;70:306-14.

21. Bolonaki I, Kotsakis A, Papadimitraki E, Aggouraki D, Konsolakis G, Vagia A, et al. Vaccination of Patients With Advanced Non-Small-Cell Lung Cancer With an Optimized Cryptic Human Telomerase Reverse Transcriptase Peptide. Journal of Clinical Oncology. 2007;25:2727-34.

22. Chiappori AA, Kolevska T, Spigel DR, Hager S, Rarick M, Gadgeel S, et al. A randomized phase II study of the telomerase inhibitor imetelstat as maintenance therapy for advanced non-small-cell lung cancer. Annals of Oncology. 2015;26:354-62.

23. Hemann MT, Strong MA, Hao L-Y, Greider CW. The Shortest Telomere, Not Average Telomere Length, Is Critical for Cell Viability and Chromosome Stability. Cell. 2001;107:67-77.

24. Ouellette MM, Liao M, Herbert BS, Johnson M, Holt SE, Liss HS, et al. Subsenescent telomere lengths in fibroblasts immortalized by limiting amounts of telomerase. J Biol Chem. 2000;275:10072-6.

\section{Figures}


Fig. 1

A

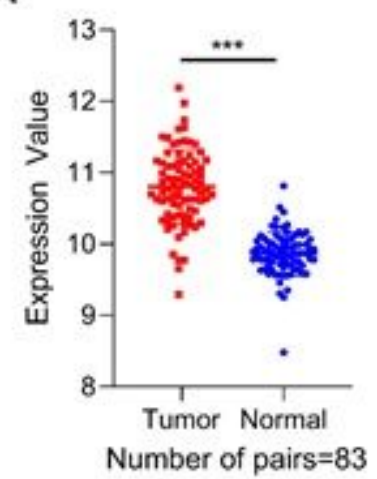

D

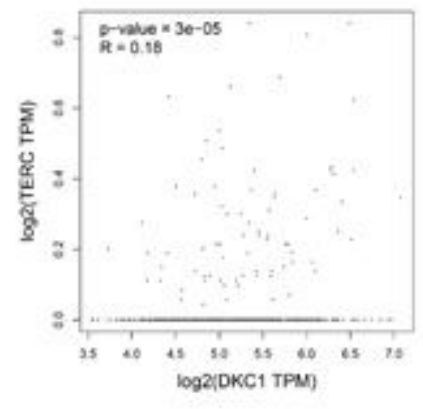

G

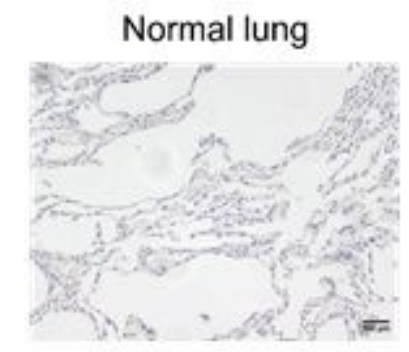

B

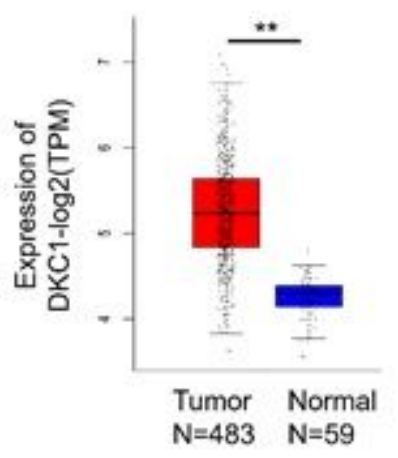

E

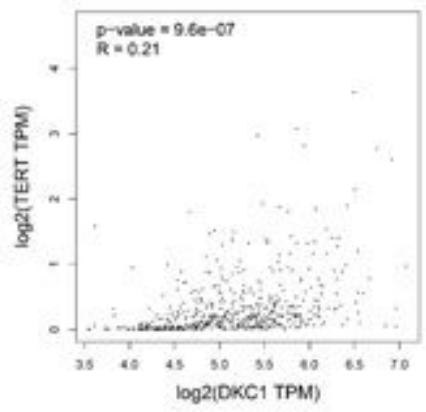

Low DKC1

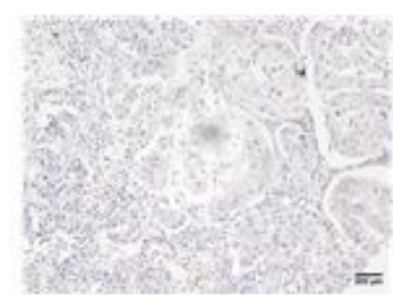

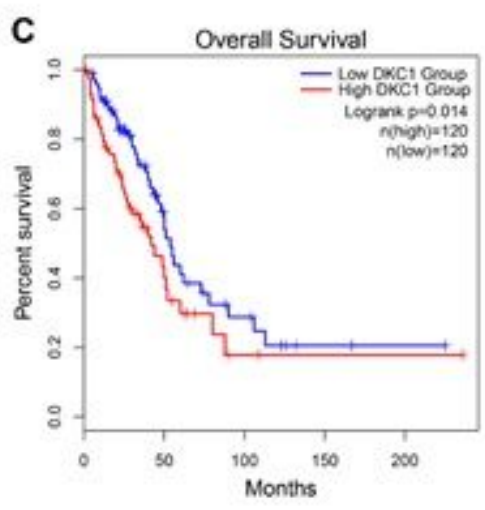

F

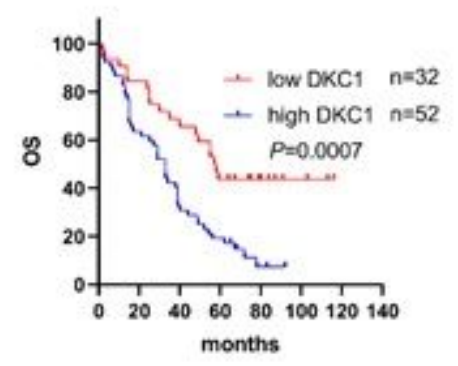

\section{Figure 1}

High expression of DKC1 in LUAD predicts poor prognosis. a The expression level of DKC1 in 83 LUAD tissues and paired normal tissues from the GEO database (GSE75037). b The expression level of DKC1 in LUAD and normal tissues from the TCGA database. c Kaplan-Meier analysis of overall survival (OS) of LUAD patients according to DKC1 level from the TCGA LUAD database. $d$, e Correlation analysis between the DKC1 level and the TERC abundance or the TERT abundance in the TCGA LUAD database. $\mathrm{f}, \mathrm{g}$ Immunohistochemistry (IHC) analysis of DKC1 expression in LUAD tissue microarray. $f$ Kaplan-Meier analysis of OS of LUAD patients according to the DKC1 expression. g Representative IHC staining of DKC1 in the normal lung tissue, LUAD tissues high DKC1 expression and low DKC1 expression. Scale bar: $200 \mu \mathrm{m} .{ }^{*} \mathrm{P}<0.01, * * * \mathrm{P}<0.0001$. 
Fig. 2

A

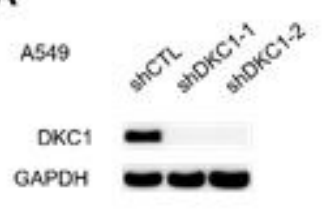

B
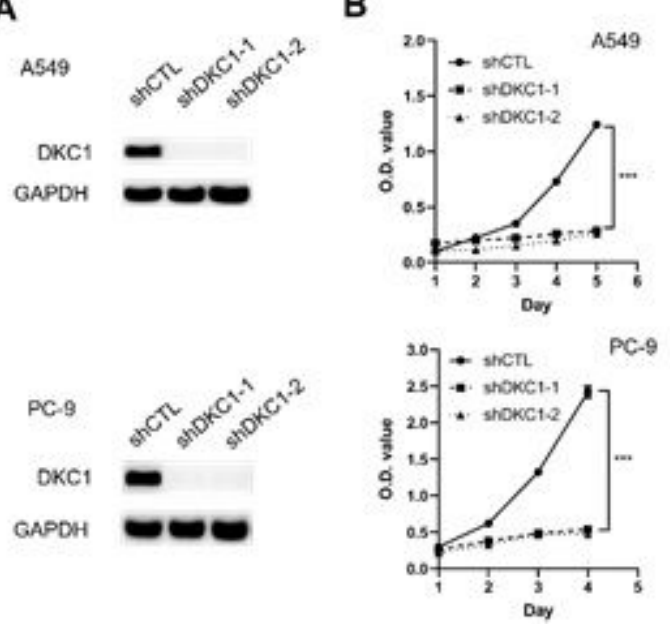

C
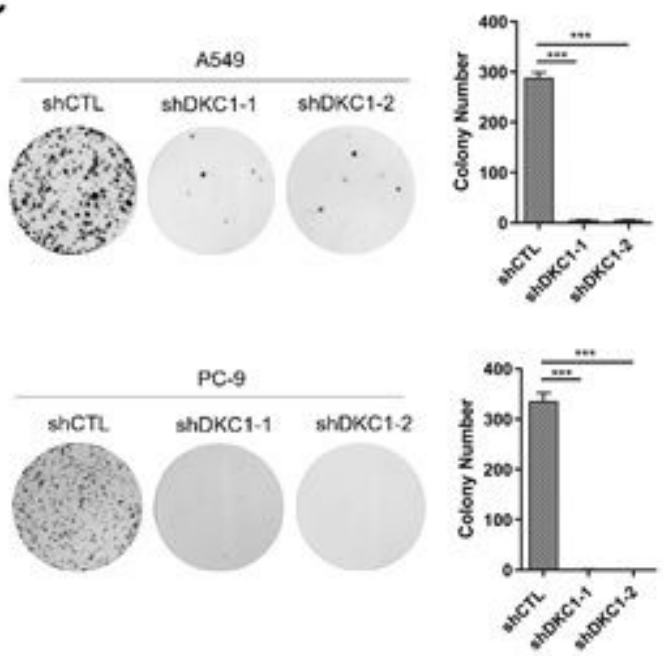

D
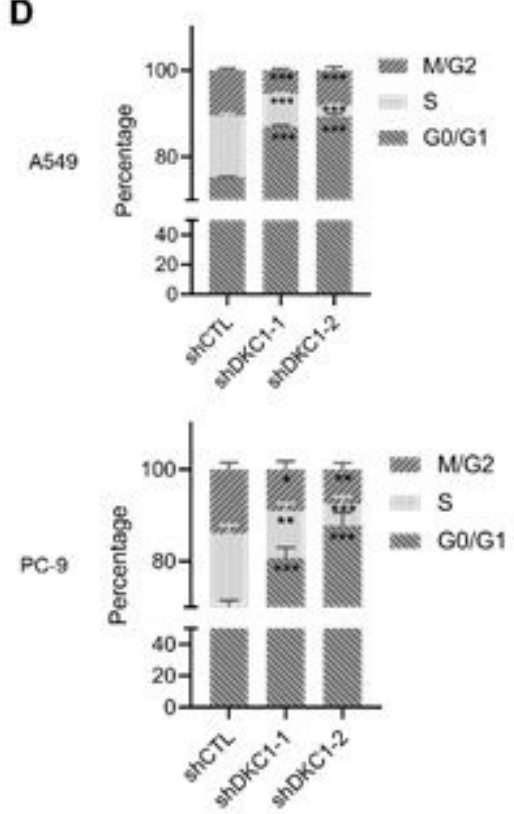

E
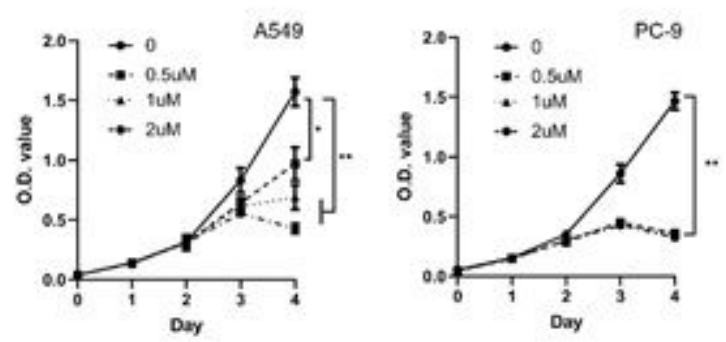

F

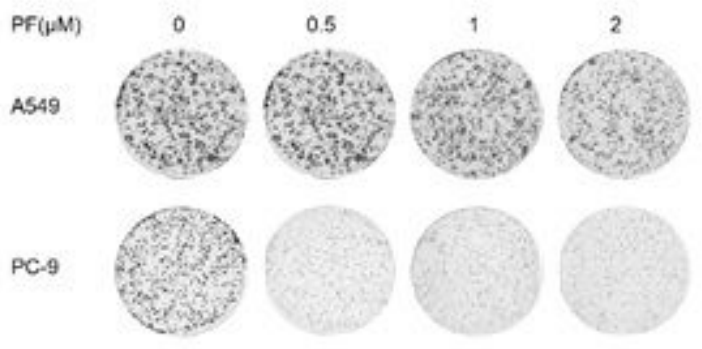

Figure 2

DKC1 knockdown inhibits LUAD cell proliferation. a Expression of DKC1 in LUAD cell lines (A549 and PC9) after lentiviral transduction of shRNA against DKC1 or nonspecific control. b Cell growth curve of LUAD cell lines (A549 and PC-9) after DKC1 knockdown. c Colony formation assays of DKC1 knockdown and control cells. The left panel shows representative colony formation images; the right panel shows a bar graph of the number of colonies per well (mean \pm SD). d Cell cycle assays of DKC1 knockdown and control A549 and PC-9 cells. e, f The effect of DKC1 inhibitor pyrazofurin on the growth and colony formation of A549 and PC-9 cells. ${ }^{\star} P<0.05,{ }^{\star *} P<0.01, * \star * P<0.0001$. 
Fig. 3

A

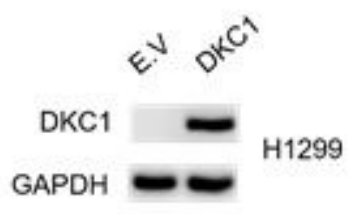

C

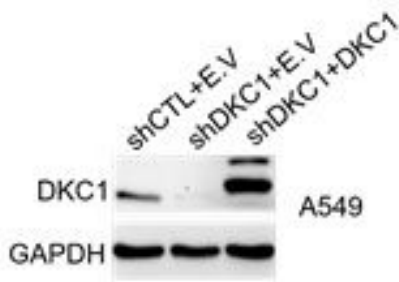

D

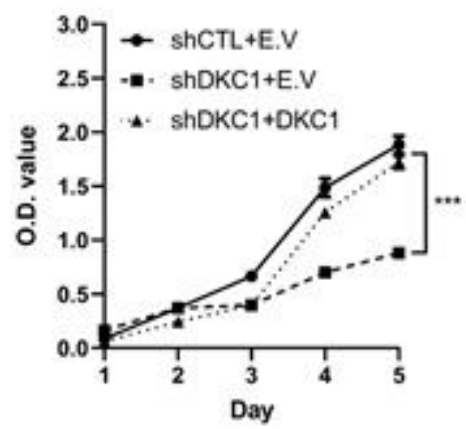

B

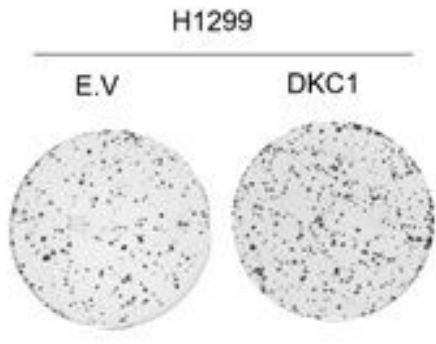

E
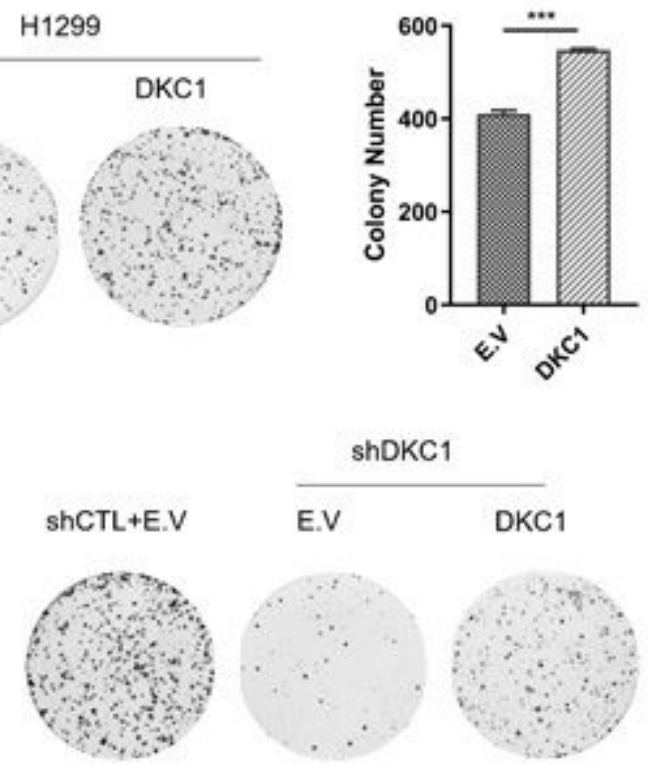

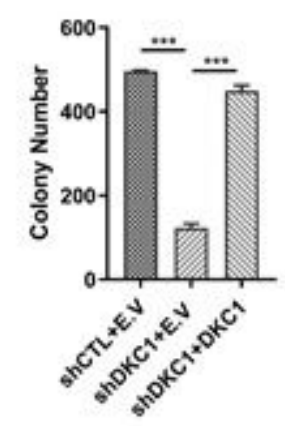

Figure 3

Ectopic expression of DKC1 accelerates LUAD cell proliferation. a Expression of DKC1 in NCI-H1299 cells after enforced expression of DKC1. b Colony formation assays of DKC1 overexpressed NCI-H1299 cells and control cells with a bar graph of the number of colonies per well (mean \pm SD). c Immunoblot detecting DKC1 expression in control and DKC1 knockdown A549 cells with ectopic expression of DKC1. d Cell growth curve of DKC1 knockdown A549 cells with ectopic expression of DKC1. e Colony formation assays of DKC1 knockdown A549 cells with ectopic expression of DKC1 with a bar graph of the number of colonies per well (mean $\pm \mathrm{SD}$ ). ${ }^{\star * *} \mathrm{P}<0.0001$. 
Fig. 4
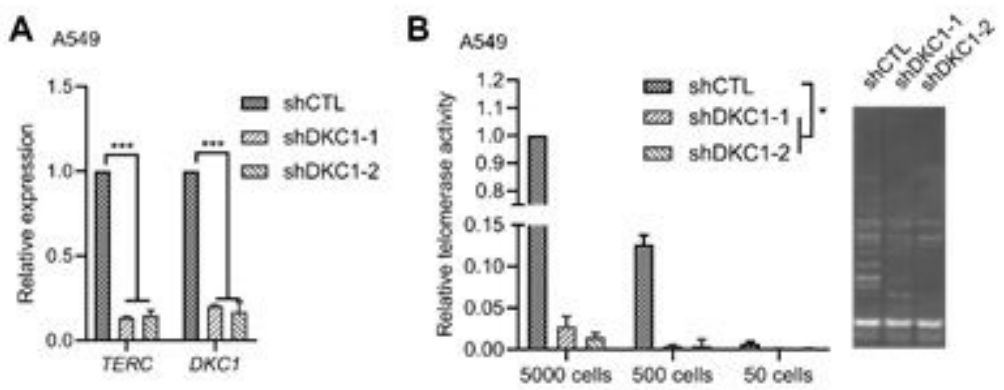

C As49
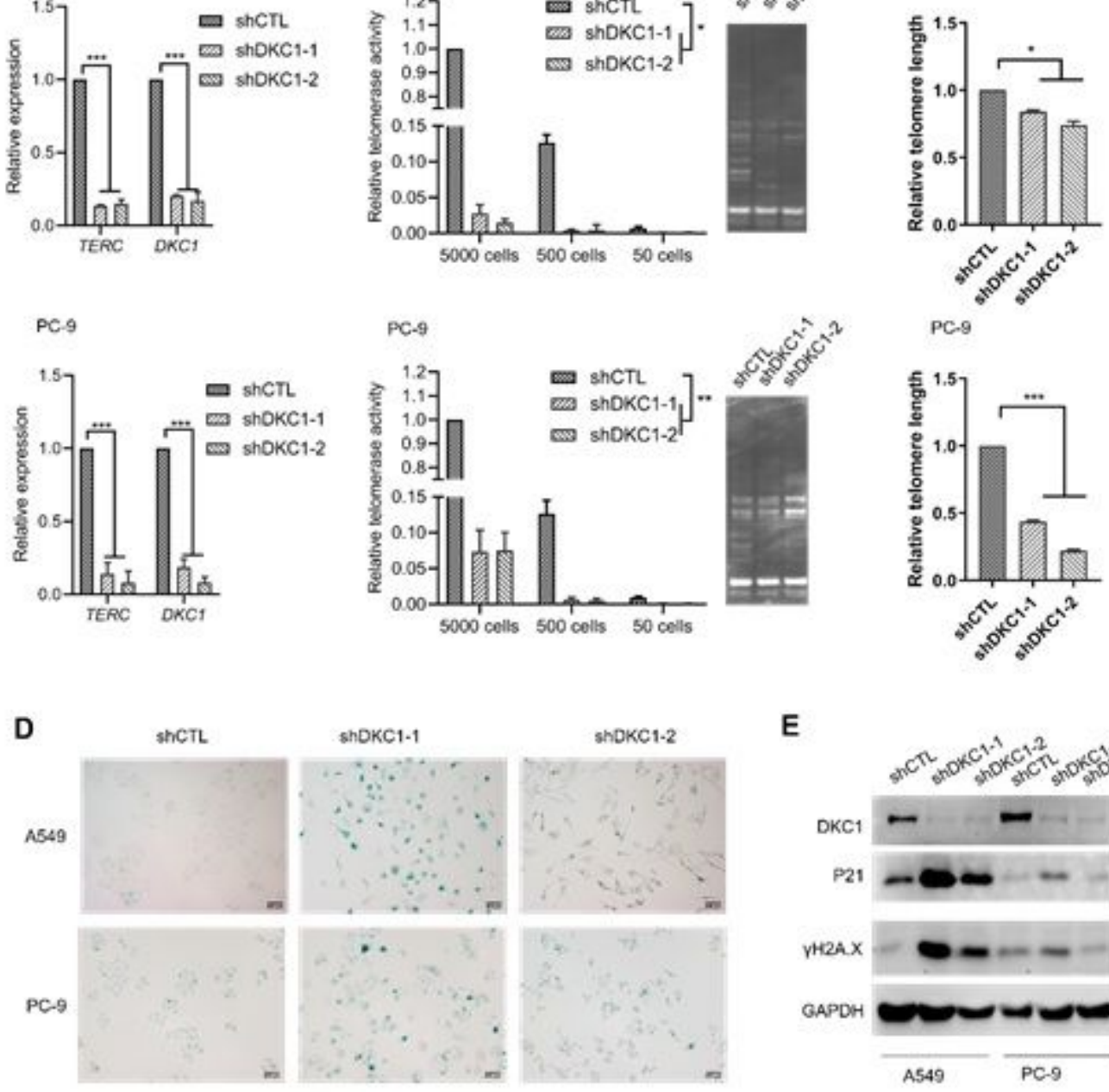

shDKC1-1

$\operatorname{shDKC1-2}$

$E$

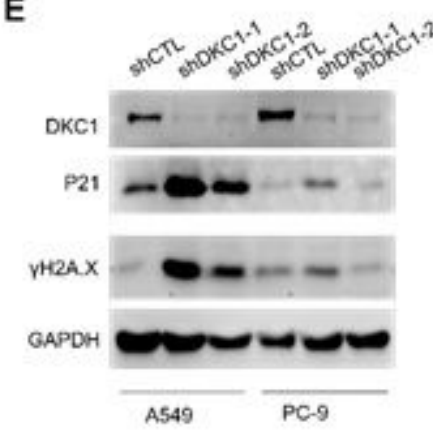

Figure 4

DKC1 downregulation induced telomere-related cell senescence and apoptosis. a qPCR detecting mRNA levels of TERC and DKC1 in DKC1 knockdown A549 and PC-9 cells. b qPCR-based Telomerase Repeated Amplification Protocol (qTRAP) analyses for telomerase activity. The left panel shows the relative telomerase activity of DKC1 knockdown A549 and PC-9 cells (5000 cells, 500 cells and 50 cells). The right panel shows the gel image of TRAP assay on 5000 cells. c qPCR-based telomere length assessment of control and DKC1 silenced A549 and PC- 9 cells. d Senescence-associated- $\beta$-galactosidase staining of

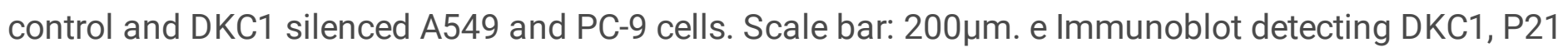
and $\mathrm{YH}_{2} \mathrm{~A}$.X expression in control and DKC1 silenced A549 and PC-9 cells. ${ }^{\star} P<0.05,{ }^{\star} \times P<0.01$, $\star \star \star P<0.0001$. 
Fig. 5
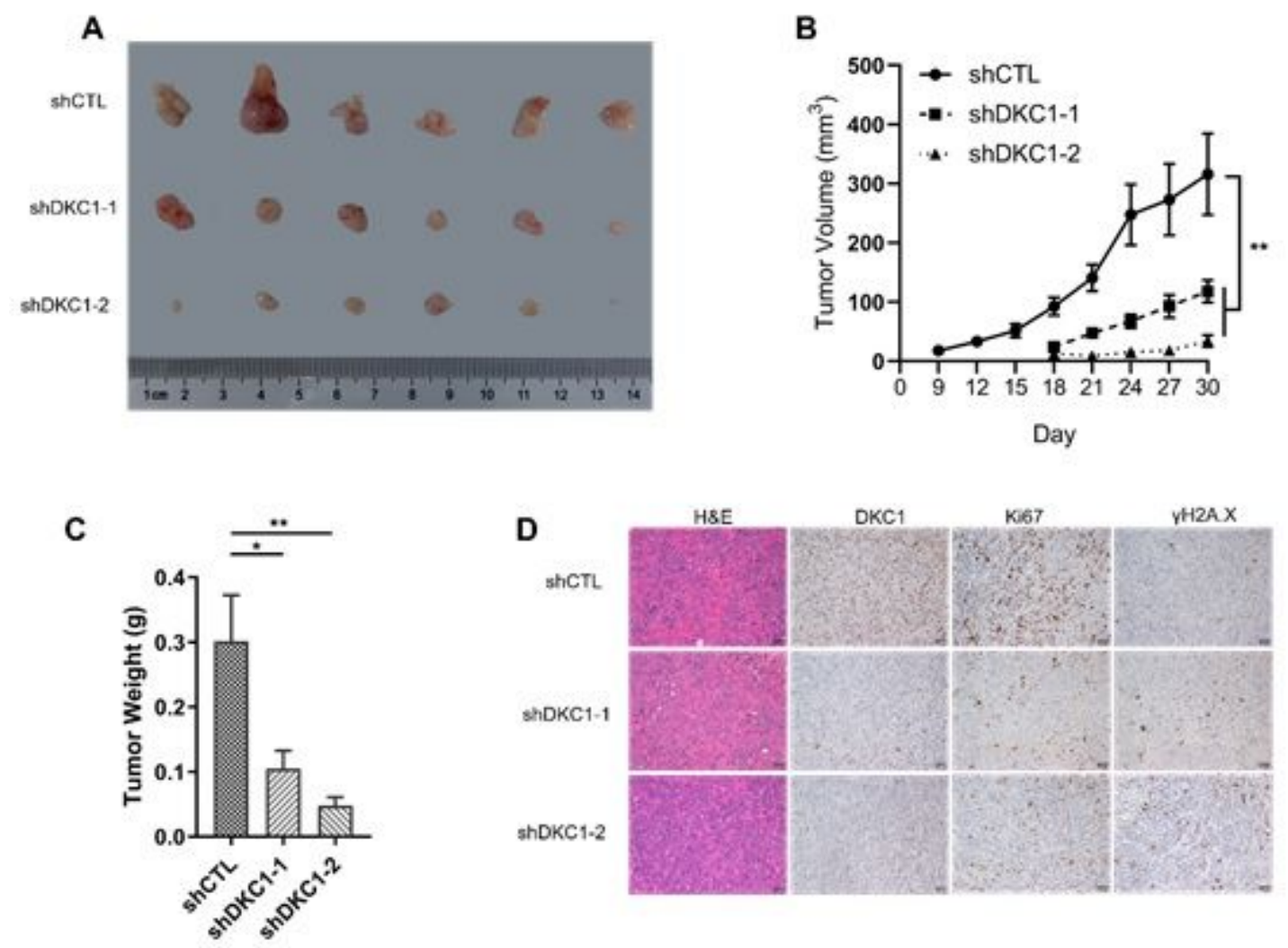

\section{Figure 5}

DKC1 knockdown inhibits LUAD proliferation in vivo. a Images of control and DKC1 knockdown PC-9 xenograft tumors 30 days after subcutaneous inoculation. b Volumes of control and DKC1 knockdown PC-9 xenograft tumors recorded at the indicated times. c Weights of control and DKC1 knockdown PC-9 xenograft tumors 30 days after inoculation. $d \mathrm{H} \& E$ and IHC staining of DKC1, Ki67 and $\mathrm{YH} 2 \mathrm{~A}$.X in control and DKC1 knockdown PC-9 xenograft tumor. Scale bar: $50 \mu \mathrm{m} .{ }^{*} \mathrm{P}<0.05,{ }^{\star *} \mathrm{P}<0.01$. 